\title{
Increased Long-Term Mortality in Healthcare-Acquired Pneumonia: Another Short-Term Lease on Life?
}

\author{
Ioannis Pneumatikos ${ }^{a}$ Demosthenes Bouros ${ }^{b}$ \\ Departments of a Critical Care and beneumonology, Medical School, Democritus University of Thrace, \\ and University Hospital of Alexandroupolis, Alexandroupolis, Greece
}

In recent years, changes in the healthcare system have shifted a considerable part of patient care from hospitals to the community. As a result, the traditional distinction between community- and hospital-acquired infections has become less clear. Infections occurring among outpatients in contact with the healthcare system have been termed 'healthcare-associated infections' [1,2]. Although limited data are available, 'healthcare-associated pneumonia' (HCAP) has recently been proposed as a new entity of pneumonia based on the following criteria: pneumonia occurring in patients with prior hospitalization in an acute care facility for $\geq 2$ days within 90 days before the onset of HCAP; residence in a nursing home or longterm care facility; recent receipt of intravenous antibiotic therapy, chemotherapy, or wound care, within 30 days before infection with HCAP; or attending a hospital or dialysis clinic [3].

An important distinction of HCAP, attributed to the above-described risk factors, is that the offending pathogens are often multidrug-resistant bacteria requiring, therefore, an initial treatment similar to that for hospitalacquired pneumonia (HAP) and ventilator-associated pneumonia (VAP), which also differentiates HCAP from community-acquired pneumonia (CAP) [4]. Patients hospitalized with HCAP have been shown to have more severe disease, longer hospital stay, and higher mortality rates than CAP [5-7].

\section{KARGER}

Fax +4161306 1234 E-Mail karger@karger.ch www.karger.com (c) 2009 S. Karger AG, Basel

0025-7931/10/0792-0089\$26.00/0

Accessible online at:

www.karger.com/res
In this issue of Respiration Cecere et al. [8], by prospectively enrolling patients and utilizing social security data to vigorously review death records, were able to confirm the long-term survival status of 457 patients categorized as having either HCAP (164 patients) or CAP (293 patients). Despite its limitations (a single-institution not a controlled study, retrospective identification of HCAP, and possible underestimation of death rate for homeless patients due to their common movement outside the state), this study is unique for two reasons: first, because the majority $(73 \%)$ of patients in this cohort were under the age of 65 at the time of admission, and second, it is the first to compare the long-term outcomes of patients admitted for HCAP with those admitted for CAP. Overall, Cecere et al. found that hospitalization for pneumonia was associated with a standard mortality ratio (SMR) of 6.0 , representing a 6 -fold increase over what would be expected in a group of patients of the same age and gender. This difference was more significant in patients admitted with HCAP than those with CAP (SMR 9.9 and 3.9, respectively). Among other risk factors independently associated with death in the multivariate analysis (i.e. age $>65$ years, presence of HIV infection before admission, use of immunosuppressant drugs other than prednisone, 'high-risk' pneumonia upon presentation, and cardiovascular disease), a diagnosis of HCAP and, to a lesser degree, of CAP was also found to be an independent pre-

Prof. Demosthenes Bouros, MD, PhD

Department of Pneumonology

Medical School, Democritus University of Thrace

GR-68100 Alexandroupolis (Greece)

Tel./Fax +30 25510 76106, E-Mail bouros@ med.duth.gr 
dictor of increased long-term mortality (only $24 \%$ survival rate at 5 years after discharge for HCAP patients $>65$ years and $47 \%$ for those $<65$ years). The socioeconomic characteristics of this relatively young patient population, such as abuse of alcohol, intravenous drug use, and homelessness, were not independently associated with an increased risk of death.

The study of Cecere and coworkers is the first to clearly show that hospitalization for HCAP may per se be a marker for poor long-term survival, even in patients who are under the age of 65 and are HIV-negative at the time of admission. This is also true for elderly patients hospitalized for CAP. Long-term mortality following an episode of CAP has been the subject of a number of reports which have shown an independent correlation between CAP in elderly patients and increased mortality [9-14]. The question is why? There is no clear explanation for this association. Yende et al. [11] examined 1- and 5-year mortality in 106 patients hospitalized with CAP and speculated that hospitalization with CAP triggers pathophysiologic processes that persist beyond recovery, possibly due to increased concentrations of proinflamma- tory cytokines or persistent abnormalities in the innate immune response. Johnstone et al. [12], describing the long-term mortality of patients in a large prospective population-based cohort enrolling 3,415 adults with CAP, suggest that it may be more likely that hospitalization for pneumonia is a more general surrogate measure for physiologic compromise or frailty.

Regarding the poor long-term survival of patients with HCAP reported in the study by Cecere et al., some points should be emphasized: HCAP is increasingly recognized as a heterogeneous disease; accordingly, the criteria employed for its designation are not entirely specific yet, leading to the misclassification of several patients [13]. Furthermore, the time of prior hospitalization as a risk factor for HCAP is not entirely clear. For example, Micek et al. [6] found that hospitalization up to 1 year prior to the onset of HCAP was important.

In conclusion, additional prospective studies are needed for better identification of patients with HCAP in order to refine the risk factors or predictors of mortality in this specific patient population.

\section{References}

1 Friedman ND, Kaye KS, Stout JE, McGarry SA, Trivette SL, Briggs JP, et al: Health careassociated bloodstream infections in adults: a reason to change the accepted definition of community-acquired infections. Ann Intern Med 2002;137:791-797.

2 Lujan M, Gallego M, Rello J: Healthcare-associated infections. A useful concept? Curr Opin Crit Care 2009;15:419-424.

-3 American Thoracic Society, Infectious Diseases Society of America: Guidelines for the management of adults with hospital-acquired, ventilator-associated, and healthcare-associated pneumonia. Am J Respir Crit Care Med 2005;171:388-416.

4 Kollef MH, Shorr A, Tabak YP, et al: Epidemiology and outcomes of health-care-associated pneumonia: results from a large US database of culture-positive pneumonia. Chest 2005;128:3854-3862.

5 Carratala J, Mykietiuk A, Fernandez-Sabe N, et al: Heath care-associated pneumonia requiring hospital admission: epidemiology, antibiotic therapy and clinical outcomes. Arch Intern Med 2007;167:1393-1399.
-6 Micek ST, Kollef KE, Reichley RM, et al: Health care-associated pneumonia and community-acquired pneumonia: a single-center experience. Antimicrob Agents Chemother 2007;51:3568-3573.

7 Venditti M, Falcone M, Corrao S, Licata G, Serra P; Study Group of the Italian Society of Internal Medicine: Outcomes of patients hospitalized with community-acquired, health care-associated, and hospital-acquired pneumonia. Ann Intern Med 2009; 150:19-26.

8 Cecere LM, Rubenfeld GD, Park DR, et al: Long-term survival after hospitalization for community acquired and healthcareassociated pneumonia. Respiration 2010;79: 128-136.

-9 Koivula I, Sten M, Makela P: Prognosis after community-acquired pneumonia in the elderly: a population-based 12-year follow-up study. Arch Intern Med 1999;159:15501555.

10 Kaplan V, Clermont G, Griffin MF, et al: Pneumonia: still the old man's friend? Arch Intern Med 2003;163:317-323.
- 11 Yende S, Angus DC, Ali IS, Somes G, Newman $A B$, Bauer D, Garcia M, Harris TB, Kritchevsky SB: Influence of comorbid conditions on long-term mortality after pneumonia in older people. J Am Geriatr Soc 2007;55:518-525.

12 Johnstone J, Eurich DT, Majumdar SR, Jin Y, Marrie TJ: Long-term morbidity and mortality after hospitalization with communityacquired pneumonia: a population-based cohort study. Medicine (Baltimore) 2008;87: 329-334.

13 Brito V, Niederman MS: Healthcare-associated pneumonia is a heterogeneous disease, and all patients do not need the same broadspectrum antibiotic therapy as complex nosocomial pneumonia. Curr Opin Infect Dis 2009;22:316-325.

14 Kofteridis DP, Papadakis JA, Bouros D, et al: Nosocomial lower respiratory tract infections: prevalence and risk factors in 14 Greek hospitals. Eur J Clin Microbiol Infect Dis 2004;23:888-891. 